\title{
Exploratory multivariate analysis of the effect of fatty fish consumption and medicinal use on heart rate and heart rate variability data
}

\section{Bjørn Grung ${ }^{1}$ *, Anita L. Hansen ${ }^{2,3}$, Mari Berg $^{1}$, Maria P. Møen-Knudseth $^{1}$, Gina Olson ${ }^{4}$, David Thornton $^{4}$, Lisbeth Dahl ${ }^{5}$ and Julian F. Thayer ${ }^{6}$}

${ }^{1}$ Department of Chemistry, University of Bergen, Bergen, Norway

${ }^{2}$ Department of Psychosocial Science, University of Bergen, Bergen, Norway

${ }^{3}$ Centre for Research and Education in Forensic Psychiatry, Haukeland University Hospital, Bergen, Norway

${ }^{4}$ Sand Ridge Secure Treatment Centre, Mauston, WI, USA

${ }^{5}$ National Institute of Nutrition and Seafood Research, Bergen, Norway

${ }^{6}$ Department of Psychology, The Ohio State University, Columbus, OH, USA

\section{Edited by:}

Andrew Kemp, Universidade de São

Paulo, Brazil

\section{Reviewed by:}

Daniel S. Quintana, University of Oslo, Norway

Kathleen M. Gustafson, University of Kansas Medical Center, USA

\section{*Correspondence:}

Bjørn Grung, Department of Chemistry, University of Bergen, Allégaten 41, 5007 Bergen, Norway e-mail: bjorn.grung@kj.uib.no
The overall aim of the present study was to explore the relationship between medicinal use and fatty fish consumption on heart rate variability (HRV) and heart rate (HR) in a group of forensic inpatients on a variety of medications. A total of 49 forensic inpatients, randomly assigned to a fish group $(n=27)$ or a control group $(n=22)$ were included in the present study. Before and by the end of the food intervention period HR and HRV were measured during an experimental test procedure. An additional aim of this paper is to show how multivariate data analysis can highlight differences and similarities between the groups, thus being a valuable addition to traditional statistical hypothesis testing. The results indicate that fish consumption may have a positive effect on both HR and HRV regardless of medication, but that the influence of medication is strong enough to mask the true effect of fish consumption. Without correcting for medication, the fish group and control group become indistinguishable $(p=0.0794$, Cohen's $d=0.60)$. The effect of medication is demonstrated by establishing a multivariate regression model that estimates $\mathrm{HR}$ and $\mathrm{HRV}$ in a recovery phase based on $\mathrm{HR}$ and $\mathrm{HRV}$ data recorded during psychological tests. The model performance is excellent for HR data, but yields poor results for HRV when employed on participants undergoing the more severe medical treatments. This indicates that the HRV behavior of this group is very different from that of the participants on no or lower level of medication. When focusing on the participants on a constant medication regime, a substantial improvement in HRV and HR for the fish group compared to the control group is indicated by a principal component analysis and $t$-tests $(p=0.00029$, Cohen's $d=2.72$ ). In a group of psychiatric inpatients characterized by severe mental health problems consuming different kinds of medication, the fish diet improved HR and $H R V$, indices of both emotional regulation and physical health.

Keywords: heart rate, heart rate variability, fatty fish, medicine, multivariate data analysis

\section{INTRODUCTION}

Psychiatric inpatients are often on a variety of medications, and it is known that the life expectancy for this group is lower than the expectancy for the general population (Nome and Holsten, 2012). Studies have also shown that people suffering from psychiatric disorders often have deficiencies in key nutrients such as omega-3 fatty acids and vitamin D (Partonen, 1998; Perica and Delas, 2011). Diet has shown to have a profound effect on both physical health, e.g., cardiovascular diseases (CVDs; Van Horn et al., 2008), and the mental health (Timonen et al., 2004). Self-reporting survey studies and nutrient supplement studies dominate this research field, due to the difficulties in conducting a well-controlled diet intervention study. Because of the low life expectancy in psychiatric inpatients on a variety of medications more knowledge about effective health care interventions is needed. Due to the fact that fatty fish is an important source of essential fatty acids and vitamin D, nutrients important for both physical and mental health (Lansdowne and Provost, 1998; Christensen et al., 2001a,b; Ross et al., 2007; Dobnig et al., 2008; Giovannucci et al., 2008), results from controlled fish intervention studies may have important implications.

Although many studies report on the effects a steady diet of fatty fish may have on the physical and mental health, the number of studies examining the underlying mechanisms are much rarer. Heart rate variability (HRV), which is an objective and important index of both physical and mental health (Thayer and Lane, 2007), has been mentioned as one such mechanism. HRV is a measure of beat-to-beat changes in the heart rate (HR), and is a measure of the interplay of the sympathetic and parasympathetic branches of the autonomic nervous system. In itself HRV is a 
predictor of sudden death in patients with coronary heart disease (Kleiger et al., 2005), which makes the relationship between fatty fish consumption and HRV interesting to investigate. A reduction in HRV is regarded as an indicator of future fatal or near-fatal cardiac arrhythmia (Huikuri et al., 2009), and it is thus an important measure of an individual's physical health. Thayer and Lane developed a model of neurovisceral integration (Thayer and Lane, $2000,2007,2009)$ that connects prefrontal cortex activity with that of the heart. This theory provides a physiological explanation as to why HRV not only predicts physical, but also emotionand self-regulation. Several later studies (Hansen et al., 2003, 2004, 2009) have underpinned the model by associating HRV with executive functions, the underlying mechanism involved in emotionand self-regulation (Shimamura, 2000; Thayer and Lane, 2000). Mozaffarian etal. (2008) found that changes in various HRV parameters were related to increased fatty fish intake. This was done by controlling for factors such as age, gender, ethnic background, education, smoking, alcohol intake, body mass index, diabetes mellitus, prevalent coronary heart disease, $\beta$-blocker use, physical activity, and intakes of beef, pork, fried fish and total calories. However, it is not well established whether the effect of fish consumption on HRV is direct or indirect (Mozaffarian et al., 2008). It has been speculated whether serotonin may be involved in the effect (Hansen et al., 2010) due to the fact that both vitamin $\mathrm{D}$ and omega-3, which are found in fatty fish, are important for the regulation of serotonin and serotonin is further important for the regulation of HRV (Stumpf and Privette, 1989; Hibbeln et al., 2006).

The use of HRV as an indicator of mental and physical health status is not problem free. A recent paper by Quintana and Heathers (Quintana and Heathers, 2014) highlights the impact of respiration on HRV. Several external factors, such as stress, age, physical shape and sickness affect the HRV, and may thus disturb the results. CVD may of course disturb the beat pattern of the heart, and persons suffering from depression tend to have lower HRV than the normal population (Kemp et al., 2010). There is a strong link between depression and CVDs, as a substantial number of CVD patients suffer from depression (Carney et al., 1987; Gonzalez et al., 1996), and persons diagnosed with depression run a higher risk of certain CVDs (Anda et al., 1993; Barefoot et al., 1996; Pratt et al., 1996; Penninx et al., 2001). Another major influence on HRV is medication. Blood pressure medications (Bonaduce et al., 1997; Pinar et al., 1998), cholesterol medication (Riahi etal., 2002; Welzig et al., 2003; Gomes et al., 2010), $\beta$ (Cook et al., 1991; Mølgaard et al., 1993; Niemelä et al., 1994; Sandrone et al., 1994; Ebbehøj et al., 2002) - and $\alpha$ - blockers and ACE inhibitors (Guedon-Moreau et al., 1997; Shehab et al., 2008) are all examples of heart medications with either known or suspected effects on HR and HRV. Among antidepressants, the most commonly used are selective serotonin reuptake inhibitors (SSRI). Serotonin itself plays a role in the HRV regulation, and an effect of SSRI on HRV can be expected. Still, various studies come to different conclusions as to whether SSRI influences HRV. In their recent review and meta-analysis, Kemp et al. (2010), concluded that some antidepressants, although successful in easing the symptoms of depression, did not improve HRV significantly. As patients with depression have a higher risk for developing CVD, a treatment that remedies the psychological symptoms, but leaves the physiological ones untreated, may imply a lower longevity for this group. Some studies (Balogh et al., 1993; Khaykin et al., 1998) demonstrate a positive effect, whereas others (Licht et al., 2010) show a negative effect on HRV. For other types of antidepressants the situation is similar, and no consensus seems to have been arrived at (van Zyl et al., 2008) on their possible effect on HRV.

Recently it was demonstrated that fatty fish consumption improved sleep quality and resting HRV (Hansen et al., 2014a). However, in that study only the effect of high frequency (HF) HRV was investigated and other HRV parameters such as low frequency (LF) and the LF/HF ratio were not investigated. Thus, a combined effect of different HRV parameters was not investigated. Moreover, no attempts were made to investigate the effect of various medications used by the participants on their psychophysiological reactivity to different conditions, such as an experimental mild-stress procedure consisting of a baseline period (resting), a variety of different cognitive tasks taxing executive functioning and a recovery (resting) period. Executive functioning tasks require focused attention over prolonged periods of time in order to be performed accurately. Carrying out these tasks can be a stressful experience. Both increased HR and decreased HRV have been found during the performance of such tasks (Porges and Raskin, 1969). Since HRV is associated with mortality, and psychiatric inpatients on a variety of medications are known to have a lower life expectancy than the general population (Nome and Holsten, 2012), investigation of HRV reactivity to different conditions while controlling for medication may yield important information of general daily autonomic activity patterns.

A major aim of this study was to generate hypotheses concerning the effects of a fatty fish diet on the HRV and HR reactivity in a group of psychiatric patients characterized by severe mental health problems consuming many different kinds of medications. Here, we focus on heart medication and anti-depressants administered on a regular basis. In order to generate new hypotheses the investigation is carried out in an exploratory way using multivariate data analysis (Principal Component Analysis and Partial Least Squares), with focus on easily interpretable plots. This technique represents a valuable addition to the battery of statistical tests normally carried out. Because of the different findings in the literature, it was also investigated whether any effect on HRV and HR could be detected prior to intervention and assigned to the usage of anti-depressants. Finally, it was investigated whether the data indicated that users of such medication experienced a different effect of the fatty fish intake compared to the participants who did not use such medication.

\section{MATERIALS AND METHODS PARTICIPANTS}

The present study was part of a larger research project concerning the effects of fatty fish consumption (Hansen et al., 2014a), where 95 male forensic inpatients characterized by severe emotional disturbances (e.g., antisocial personality disorders, borderline personality disorders, generalized anxiety and major depression) had been randomly assigned into a Fish group and a Control group. In the current study a subset of 49 participants were suitable for an investigation of whether the intake of medications had an impact 
on the effect of the fish consumption. Details about the study progress are reported in Hansen et al. (2014a). The number of subjects in the fish group was 27 . The number of subjects in the control group was 22. During the test period five subjects withdrew from the fish group, and six from the control group. Details on then randomization procedure can be found in Hansen et al. (2014b).

\section{APPARATUS}

Physiological activity was measured by recording HR and HRV using the Actiheart System (Cambridge Neurotechnology Ltd; Brage et al., 2005), a compact lightweight device that records HR and variability of R-R inter-beat intervals. The Actiheart clips onto a single ECG electrode (M-00-S/50 Blue Sensor) with a short ECG lead to another electrode that detects the ECG signal. The Actiheart was placed on the upper chest.

The Actiheart provides interbeat intervals with a resolution of $1 \mathrm{~ms}$, which are used to calculate HR and HRV. HR was defined as the average HR in beats per minute for an analysis epoch of $1 \mathrm{~min}$. In the frequency domain, HRV was measured as absolute high frequency power (HF; $0.15-0.4 \mathrm{~Hz}$ ), absolute low frequency power (LF; $0.04-0.15 \mathrm{~Hz})$ and their ratio $(\mathrm{LF} / \mathrm{HF})$. HF and LF were derived by fast Fourier transform of the spectrum. The HF component is known to reflect primarily parasympathetic influences. However, concerning the LF there is some controversy. It has been argued that LF reflects both sympathetic and parasympathetic activity; others argue that LF reflects only sympathetic activity (Malliani et al., 1991; Appelhans and Luecken, 2006; Xhyheri et al., 2012). In the time domain, HRV was measured as the root mean of the squared successive differences (RMSSD) of the inter-beat interval. The RMSSD reflects parasympathetic activity. The data was log transformed prior to analysis (Malik and Task Force of the European Society of Cardiology the North American Society of Pacing Electrophysiology, 1996). Artifacts were manually cleaned by visual inspection using the Actiheart program.

\section{PROCEDURE}

The study protocol and all experimental procedures were approved by the Ethics Committee at the facility in Mauston, WI, USA, and were in compliance with the Helsinki declaration for research ethics. Participants were recruited by both written and oral information about the study. Thus, the patients were invited to participate in a research project concerning nutrition and mental health. The participants were informed that the purpose of the study was to investigate if nutrition (fatty fish or an alternative meal like chicken, pork, or beef) would have any effects on mental health. They were also informed that they would be randomly assigned into two groups; one group that would eat fatty fish (portion size 150-300 g) three times a week and one group that would eat meat (e.g., chicken, pork, beef) meals three times a week for a period for 6 months (September-February). For information about the fish used in the intervention study, see Hansen et al. (2014a). The participants signed an informed consent form, and they were informed about their rights to withdraw from the study at any time for any reason without penalty. Thus, the study design was fully disclosed to all participants, and all participants provided informed consent.
Prior to (July) and toward the end (February) of the intervention period the participants went through a test procedure. Since the present study is part of a larger project concerning fatty fish consumption (Hansen et al., 2014a) these procedures involved collections of various kinds of data such as fasting blood sample, sleep data, self-report questionnaires as well as an experimental test procedure measuring psychophysiological activity during exposure to different executive function tasks. In order to obtain a broad measure of reactivity, psychophysiological activity was registered for $5 \mathrm{~min}$ of baseline (rest condition), during exposure to the different executive function tasks (i.e., Iowa Gambling Task, Tower of Hanoi, and N-back tasks; 0-3 back), and during 5 min of recovery (rest condition). The length of executive tasks varies from task to task, and person to person. Thus, the HRV was registered for a total period of 50-60 min. During these periods of time, one measurement epoch lasts for $1 \mathrm{~min}$. This means that there are five measurements for baseline and recovery (five epochs of $1 \mathrm{~min}$ during a $5 \mathrm{~min}$ interval), and a varying number of measurements for the tasks. All participants were exposed to exactly the same experimental procedure before (pre) and after (post) the food intervention period. All participants were tested individually.

\section{MEDICINAL USE}

Copies of all medical records, from 1 month prior to 1 month after intervention, were available for a subset of participants. The various drugs administered were classified according to the Anatomic Therapeutic Chemical Classification System, which is controlled by the World Health Organization. For each participant, daily dosages of each pharmaceutical used continuously for at least 1 week prior to the pre-test period were registered. This was done to avoid inclusion of drugs to which the participants had not yet properly responded. The same procedure was carried out for the post-test. For the forthcoming data analysis, only categories C (cardiovascular drugs) and N06A (anti-depressants) were used actively in the analysis.

Some participants were excluded from the data analysis. Participation in the project was voluntary, and three participants withdrew for various reasons during the intervention period. Participants released from the facility or transferred to a different facility during the study were included in the analysis of the pre-test, but are of course not present in the post-data. Participants with partially missing journals were excluded from further analysis. For some participants, a substantial amount of missing data was observed in the HR and HRV data. Participants with more than $20 \%$ missing data were excluded from further analysis.

\section{THE DATA TABLES}

A data matrix was constructed from the pre-test data. It contained HR and HRV data for 49 participants. For each participant there were 40 variables recorded. These were the HR and the four HRV measures (LF, HF, LF/HF, and RMSSD) recorded during the eight stages of the experiment: baseline, the six psychological tests and recovery. A similar matrix was created from the post-intervention test data. To highlight the effect of the intervention on the HR and HRV, the pre-test data was subtracted from the post-test data, 
thus yielding a matrix reflecting the change in HR and HRV measures. The resulting table is hereafter referred to as the change data. The creation of this matrix necessitated a full set of data from both before and after the intervention, and thus only 38 participants are included in this matrix. Five of the 11 participants lost to follow up belonged to the fish group, and six belonged to the control group. Data was logarithmically (base 10) transformed prior to analysis. Due to the variables being measured using different units of measurements, autoscaling was carried out whenever HR and HRV data was used in the same analysis. For analysis using only HR or HRV data, mean centering of the variables was carried out.

\section{THE DATA ANALYTICAL TOOL}

The data analytical tool used in the investigation here may be unfamiliar to many readers. Principal Component Analysis (PCA; Joliffe, 2002) and Partial Least Squares (Manne, 1987) belong to a group of methods which may be referred to as latent variable techniques. They are methods frequently employed in chemometrics and related fields. The strength of these methods comes from their ability to deal with collinear data structures, in which the variables exhibit correlations. Another advantage of these methods is the easily interpreted plots that result from such analysis, and a presentation of this is to be found in the Results section.

While a more detailed mathematical explanation can be found in, e.g., Manne (1987) and Joliffe (2002), a brief explanation is given here. Latent variable methods take a set of correlated, measured variables (like the HR and HRV data in this study) and create a new, smaller set of orthogonal latent variables. The latent variables are linear combinations of the measured variables, and may be constructed using different criteria. In PCA, the latent variables (called principal components) are constructed using the maximum variance criterion. This means that the set of principal components are the best lower-dimensional approximation of the data, and that they represent the major sources of variation in the data. This makes PCA well suited for exploratory analysis.

Just like any object (participant in our study) has a value for each measured variable, all objects have a score for each principal component. Similarly, the contribution of each measured variable to the latent variable is expressed as the variable's loading. Bivariate scatter plots of the first two score or loading vectors give the best two-dimensional presentation of the major correlation structures in the data, and reveal similarities and differences among the objects and variables in a data matrix. This soft modeling approach can be regarded as a hypothesis generator, since it visualizes relationships in the data that may otherwise go unnoticed. Latent variables can also be used to confirm or verify preconceived assumptions and hypotheses, since it is possible to carry out statistical tests on the latent variables. In the present work, the emphasis has been on data exploration, and less on quantification and hypothesis testing.

For the creation of regression models, other latent variable techniques are preferred. Rather than focusing on the variance in the predictors (the independent variables), PLS creates latent variables focusing on the covariance between the predictors and one or several response variables (the dependent variables). Because of the orthogonalization inherent in PLS, this regression technique does not suffer from the problem of collinear data that, e.g., multiple linear regression does.

The multivariate analyses were carried out using Sirius 9.0 from Pattern Recognition Systems AS, Bergen, Norway (www.prs.no).

\section{RESULTS}

Table 1 displays summary statistics for the HR and HRV data at pre- and post-test. For the executive functioning tasks (EF tasks) the average HRV and HR values for all cognitive tasks (Iowa Gambling Task, Tower of Hanoi, and N-back tasks; 0-3 back) are reported.

\section{PRE-TEST}

Plots of data are often useful conveyors of information. The data matrix from the pre-test period contains 40 measurements for each of the 49 participants. A graphical display of the information content in such a large data matrix may seem difficult to obtain. One alternative is to plot each variable separately (thus creating 40 plots), or to produce all possible combinations of bivariate plots of one variable against another. This creates 780 plots, which of course is impossible to carry out. Through PCA a two-dimensional projection capturing most of the variation in the data can be obtained, resulting in a bivariate plot to be used for interpretation. For this particular data set, a two component (two latent variables) PCA model captures nearly $70 \%$ of the variance in the data. A bivariate plot of these two latent variables is shown in Figure 1, and it is referred to as a score plot. Since the axes in Figure 1 are latent variables, information from all 40 measured variables is used to create this plot showing information on all 49 participants. This multivariate nature of the axes makes the plot an excellent tool for detecting relationships among the individual participants. In Figure 1, members of the fish group are plotted in blue, while red has been used for the control group. This information has only been used to color code the plots, and is not included in the data material.

There is no discernable trace of any grouping of the samples; the future fish and control group members are spread evenly throughout the plot. This indicates that the randomization strategy has been successful, as there is no visible group separation prior to intervention. A $t$-test on the scores on the first principal component shows that there is no significant difference in the mean value of the score values of the fish group compared to the control group $(p=0.38$, effect size Cohen's $d=0.25)$.

By changing the color coding (but not the underlying model) clustering can be revealed. Figure 2 is the result of changing the color coding so that patients on anti-depressants are plotted in blue and those not using anti-depressants in red. The data analyzed and the resulting model is the same as in Figure 1, only the color coding has changed. Figure 2 shows that patients using anti-depressants differ from the other patients by having a lower score on the first principal component. The findings from the PCA are confirmed by a $t$-test on the mean values of the scores on the first principal component. This results in a clear identification of a difference between the groups ( $p=0.0037$, effect size Cohen's $d=1.01$ ).

While the score plot shows clustering, the corresponding loading plot is necessary to understand why groupings occur. Figure 3 
Table 1 | Mean values (M) and SD for the heart rate (HR), the root mean square of successive differences (RMSSD), low frequency power (LF), high frequency power (HF), and the LF/HF ratio for both groups during pre- and post-test.

\begin{tabular}{|c|c|c|c|c|c|c|}
\hline & \multicolumn{3}{|c|}{ Fish group } & \multicolumn{3}{|c|}{ Control group } \\
\hline & $M$ & SD & $n$ & $M$ & SD & $n$ \\
\hline \multicolumn{7}{|l|}{ Pre-test } \\
\hline Baseline HR & 72.96 & 13.15 & 27 & 72.81 & 13.13 & 22 \\
\hline Baseline RMSSD & 1.44 & 0.28 & 27 & 1.45 & 0.27 & 22 \\
\hline Baseline LF & 3.02 & 0.40 & 27 & 2.90 & 0.40 & 22 \\
\hline Baseline HF & 2.22 & 0.59 & 27 & 2.29 & 0.49 & 22 \\
\hline Baseline LF/HF & 0.82 & 0.33 & 27 & 0.64 & 0.26 & 22 \\
\hline EF tasks HR & 71.48 & 13.06 & 27 & 70.12 & 11.82 & 22 \\
\hline EF tasks RMSSD & 7.23 & 0.34 & 26 & 7.05 & 3.87 & 22 \\
\hline EF tasks LF & 2.82 & 0.59 & 26 & 2.86 & 0.37 & 22 \\
\hline EF tasks HF & 2.14 & 0.75 & 26 & 2.36 & 0.46 & 22 \\
\hline EF tasks LF/HF & 0.69 & 0.27 & 26 & 0.53 & 0.22 & 22 \\
\hline Recovery HR & 70.47 & 12.08 & 26 & 69.71 & 12.14 & 22 \\
\hline Recovery RMSSD & 1.62 & 0.31 & 27 & 1.63 & 0.27 & 22 \\
\hline Recovery LF & 3.30 & 0.43 & 27 & 3.32 & 0.39 & 22 \\
\hline Recovery HF & 2.61 & 0.61 & 27 & 2.69 & 0.46 & 22 \\
\hline Recovery LF/HF & 0.79 & 0.41 & 27 & 0.72 & 0.28 & 22 \\
\hline \multicolumn{7}{|l|}{ Post-test } \\
\hline Baseline HR & 71.08 & 12.50 & 22 & 76.11 & 9.87 & 16 \\
\hline Baseline RMSSD & 1.56 & 0.28 & 22 & 1.47 & 0.32 & 16 \\
\hline Baseline LF & 3.11 & 0.40 & 22 & 2.99 & 0.59 & 16 \\
\hline Baseline HF & 2.39 & 0.54 & 22 & 2.36 & 0.54 & 16 \\
\hline Baseline LF/HF & 0.78 & 0.40 & 22 & 0.68 & 0.17 & 16 \\
\hline EF tasks HR & 70.37 & 11.33 & 22 & 73.96 & 11.08 & 16 \\
\hline EF tasks RMSSD & 1.54 & 0.36 & 22 & 1.52 & 0.26 & 16 \\
\hline EF tasks LF & 2.92 & 0.47 & 22 & 2.81 & 0.49 & 16 \\
\hline EF tasks HF & 2.37 & 0.70 & 22 & 2.38 & 0.49 & 16 \\
\hline EF tasks LF/HF & 0.57 & 0.30 & 22 & 0.47 & 0.28 & 16 \\
\hline Recovery HR & 69.75 & 9.98 & 22 & 75.17 & 10.81 & 16 \\
\hline Recovery RMSSD & 1.63 & 0.30 & 22 & 1.49 & 0.23 & 16 \\
\hline Recovery LF & 3.26 & 0.38 & 22 & 2.97 & 0.44 & 16 \\
\hline Recovery HF & 2.61 & 0.62 & 22 & 2.44 & 0.40 & 16 \\
\hline Recovery LF/HF & 0.73 & 0.33 & 22 & 0.60 & 0.27 & 16 \\
\hline
\end{tabular}

The HRV data are log-transformed. The number of participants in each group is in the column $n$.

shows the loading plot. Again, color coding has been used. HR variables are plotted in red, and blue has been used for the RMSSD variables. The HF variables are in black, and green has been used for $\mathrm{LF}$. The $\mathrm{LF} / \mathrm{HF}$ ratio is plotted in brown.

Figure 3 shows that participants with a low score on the first principal component (the patients using anti-depressants) have a tendency to have a higher HR (red) and LF/HF (brown) compared to the rest of the patients.

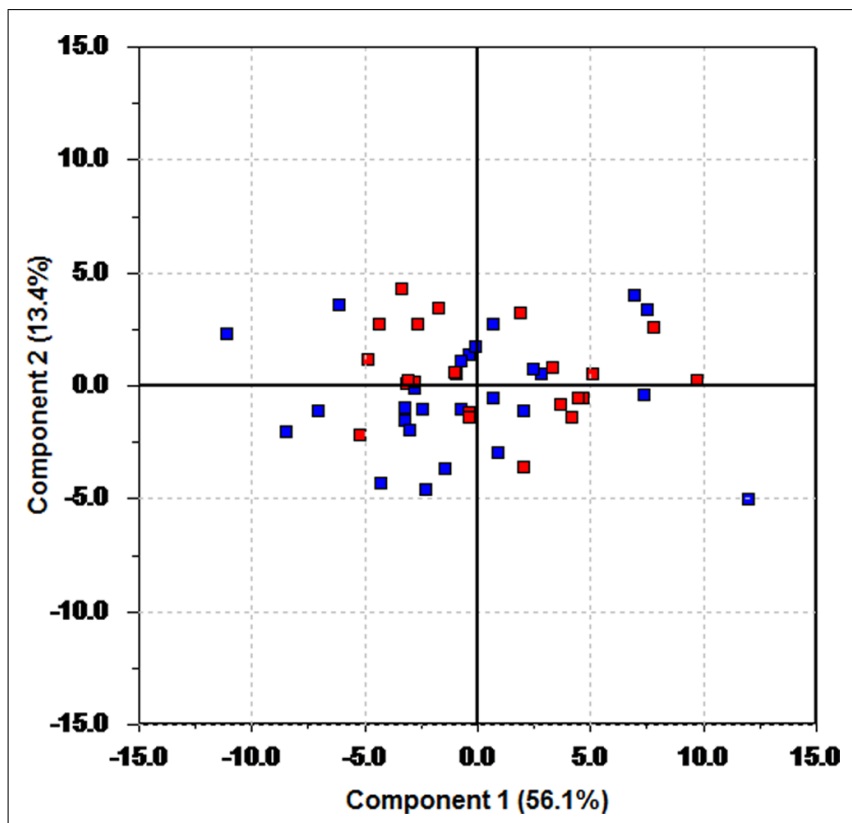

FIGURE 1 | Score plot of the first two principal components of the pre-test data. Blue color is used for the fish group, and red for the control group.

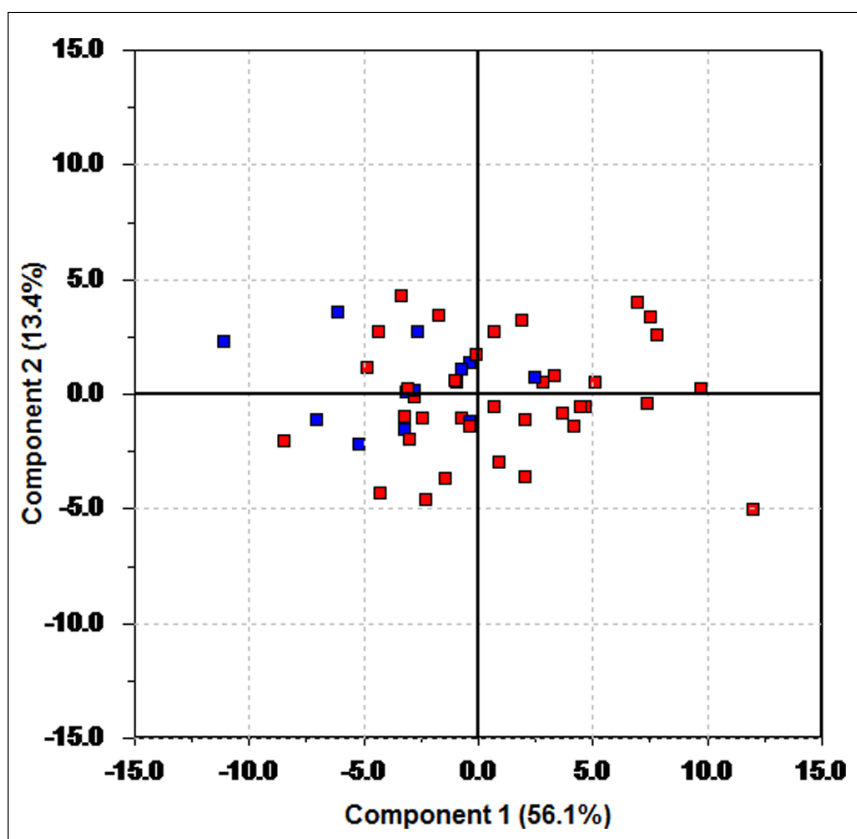

FIGURE 2 | Score plot of the first two principal components of the pre-test data. Blue color is used for participants using anti-depressants, and red for those without such usage.

The recovery phase is an indicator on how the body adjusts to the normal situation after a period of mild stress. It was thought to be of interest to see if the patients on anti-depressants responded differently during recovery. This was done by creating PLS regression models that modeled recovery behavior as a function of behavior during baseline and the test period. Separate models 


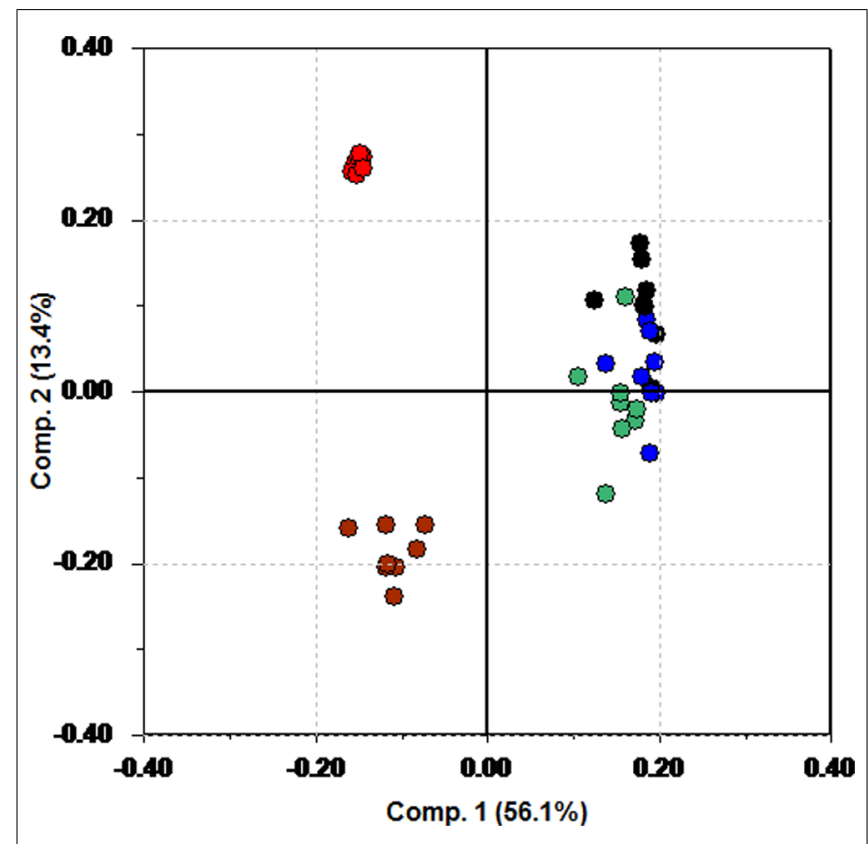

FIGURE 3 | Loading plot from PCA of the pre-test data. Red is HR, blue is RMSSD, black is HF, green is LF, and brown is used for LF/HF.

were created for HR and each of the individual HRV measures. In Figure 4A, a plot of modeled recovery versus actual recovery for HR is shown. Again, blue color is used to denote participants using anti-depressants. The blue line represents perfect match between modeled and actual response. Figure 4A shows a good correspondence between measured and estimated HR for the whole group. The HR behavior during recovery is possible to estimate for all test participants, regardless of medication use.

This situation changes when looking at some of the HRV measures. Figure 4B illustrates the situation for RMSSD. For several participants the model performance is poor. For five of the participants (to the right), the actual RMSSD is far higher than expected based on the model. There is also one participant (to the left) with a measured RMSSD far lower than expected according to the model. This interesting result shows that this group of patients, all using anti-depressants, undergoes a different recovery phase with regards to RMSSD, compared to the other patients. A similar behavior is experienced when studying LF, but not for HF, or LF/HF.

\section{THE CHANGE DATA}

The most important data table investigated in this work was created by subtracting the pre-test data from the post-test data. This was done to focus on each participant's change in HR and HRV during the intervention. The score plot from PCA on this data set (not shown), shows little sign of discrimination between the fish and control group. A $t$-test on the scores confirms this $(p=0.0794$, Cohen's $d=0.60$ ).

However, clear separation between the fish and control group can be achieved by controlling for medication. In Figure 5, two score plots from PCA on a subset of participants free from heart medication and anti-depressants are shown. Again, blue represents intervention and red control. Figure 5A shows the results from a PCA on this subset of participants. The ellipses in the plot are Hotelling's $\mathrm{T}^{2}$ limits (Jackson, 1991), which can be regarded as a multivariate generalization of a $t$-test. An outlier is present in the upper right quadrant of the plot. Removal of this outlier and recalculation of the model yields the score plot in Figure 5B. Separation is clearly visible, and a $t$-test on the mean value of the scores of the first principal component ( $p=0.00029$, Cohen's $d=2.72$ ) show that the two groups are different, and that the fish group has undergone a change in HR and HRV different to the intervention group.

A closer look at the status of subjects in the fish group is presented in Figure 6. This analysis was performed on the patients whose medication was constant throughout the intervention. Red is used to indicate the participants on constant medication, while blue is used for the ones who did not receive any medication. With the small sample size here, one should be careful with drawing strong conclusions. However, separation of the groups is evident from the score plot, as the patients not using medication is positioned more in the lower right part of the plot. This indicates a difference in response to the intervention. Still, the small sample size makes this observation less certain than the others presented in this work. We have therefore not carried out any additional statistical tests, but the plot generates the interesting hypothesis that the participants on medication respond differently to the fish diet.

The loading plot in Figure 7 illuminates why there is a separation. The same color coding is used in this loading plot as in Figure 3. HR variables are plotted in red, and blue has been used for the RMSSD variables. The HF variables are in black, and green has been used for LF. The LF/HF ratio is plotted in brown.

In the upper left region we find some of the LF, HF, and RMSSD measures, whereas larger change in HR and LF/HF measures seem to be associated with the participants not on medication.

\section{DISCUSSION}

The use of latent variables as a data analytical tool is probably new to many readers. We do not suggest that these methods should replace traditional statistical tests, but rather complement them. Latent variables, in conjunction with statistical tests, showed a successful randomization procedure, as seen in Figure 1. A priori knowledge of group membership can still be used effectively by color coding the participants and variables accordingly to highlight the information contained in the variance pattern of the data. A PCA on the change data was initially unable to separate the fish group from the control group ( $p=0.0794$, Cohen's $d=0.60)$. This is due to the criterion used to calculate the principal components. The maximum variance criterion forces the principal components to pick up the major variation sources, at the expense of leaving minor, systematic changes unaccounted for. The effect of medication was strong enough to hide the effect of the intervention. Only by controlling for medicinal use a separation of the two groups became visible in the score plots $(p=0.00029$, Cohen's $d=2.72$ ). Although there of course are huge individual HR and HRV variations due to other external factors, the intervention group in general scores higher on the first principal component. This demonstrates that whether or not a participant is 

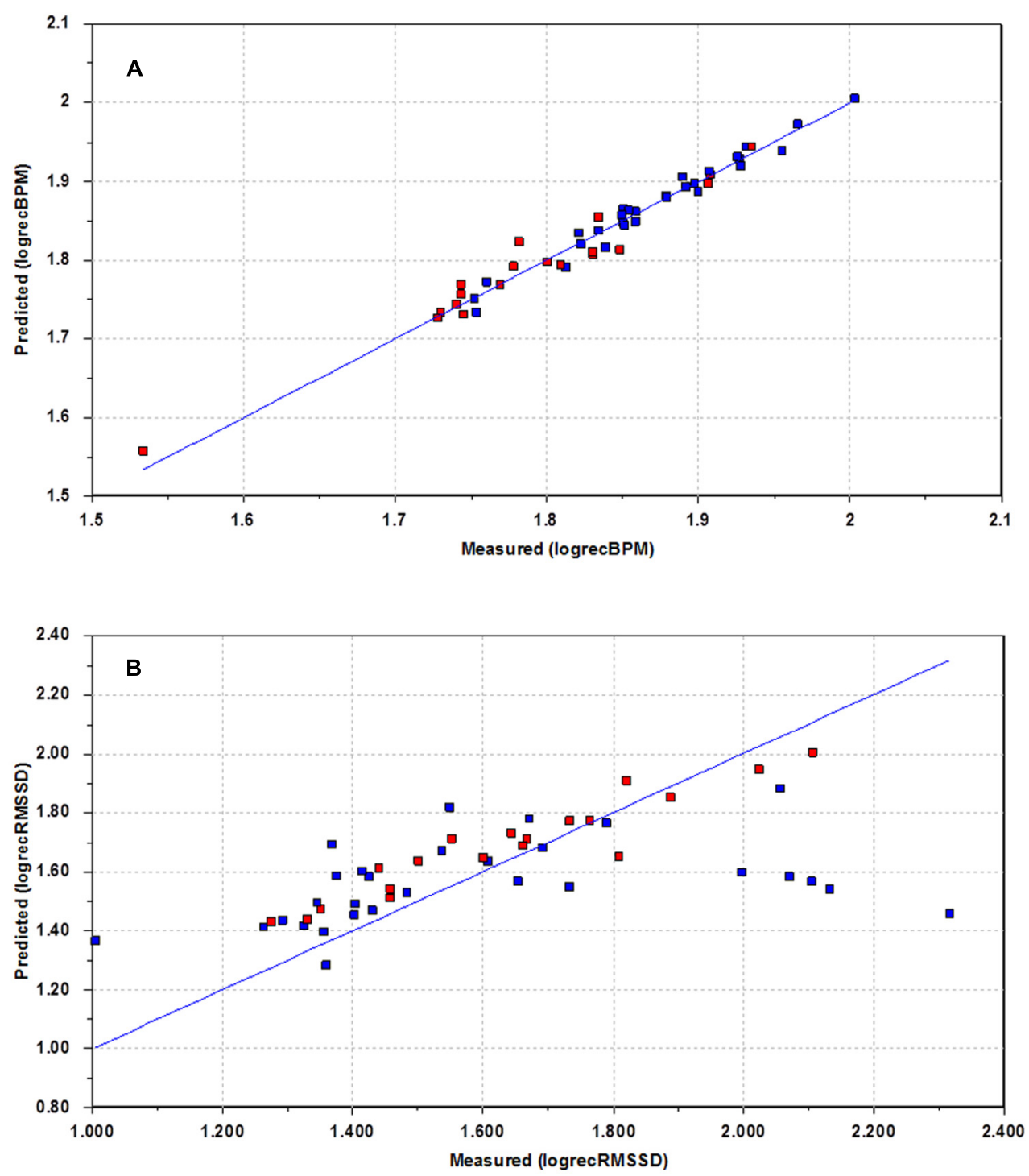

FIGURE 4 | Plots of estimated recovery versus measured recovery. Blue - uses heart medication and/or anti-depressants. Red - does not use drugs. (A) Recovery HR. (B) Recovery RMSSD.

on the fish diet is a major source of variation in the data. A similar plot (not shown here) appears if one investigates only the subset of participants, whose medication remained constant throughout the intervention period, again indicating that the introduction of fatty fish to the diet is a major source of variation once medication is controlled for.

The use of quantitative regression modeling using latent variables has been shown for parts of the recovery data. The poor performance of the model for RMSSD prediction for some of the participants indicates that the recovery phase is different for some of the participants on heavy medications. For the majority of participants the model performance is good, so an interesting question is to ask why the model fails for some participants. A closer look at the medical records reveals that the participants in question are using a variety of heart medications and anti-depressants; up to eight different drugs at the same time. Thus, participants with a higher level of medication respond differently during the recovery phase to the normal population. Five of the six participants for which the prediction fails were using statins, a cholesterol lowering drug which previously has been shown to improve HRV (Riahi et al., 2002). Four participants were using fibrates, another cholesterol lowering drug which has been reported to improve HRV (Melenovsky et al., 2003). There seems to be little consensus in the literature as to what the effects of SSRIs are on HRV (van Zyl et al., 2008; Kemp et al., 2010; Licht et al., 2010). However, a recent large independent cohort study showed that different antidepressants (e.g., tricyclic antidepressant, SSRI) were related to lower HRV (Kemp et al., 2014). The fact that the present recovery prediction model fails for the HRV data for the participants on heavy medication, again 

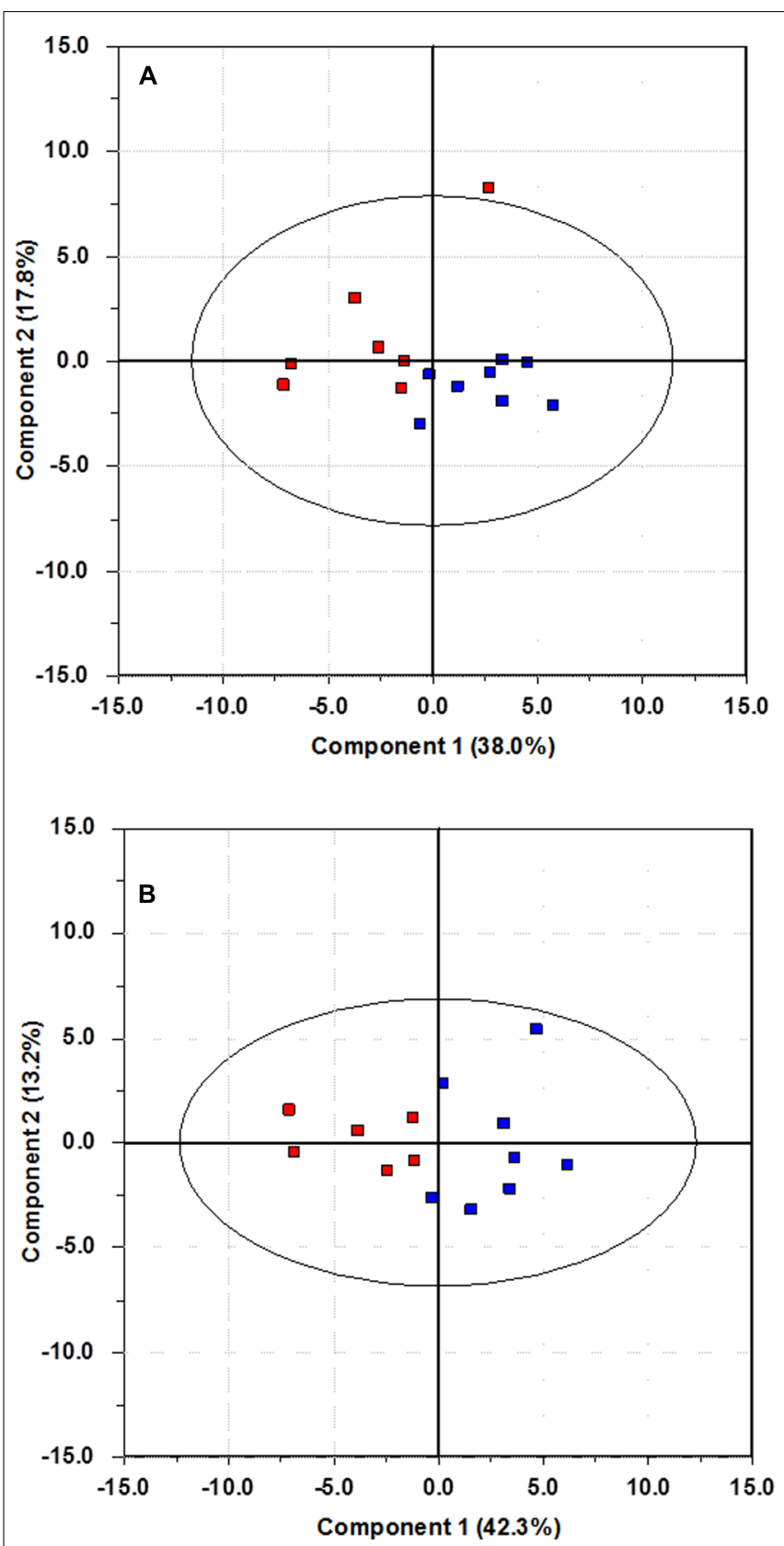

FIGURE 5 | Score plot of the first two principal components of the change data for the participants using neither heart medication nor anti-depressants. Blue color is used for the fish group, and red for the control group. (A) Outlier included. (B) Outlier excluded.

demonstrates the strong masking effect medication has on the HRV.

The participants in this study were adult male forensic inpatients. This is a group for which cognitive and executive functions deficits have been demonstrated before (Galski et al., 1990). Thus, based on the very high RMSSD during recovery in these participants on a mix of medication, one can speculate whether the level of HRV during recovery may add some information about the physiological effort invested in the task before the recovery. The

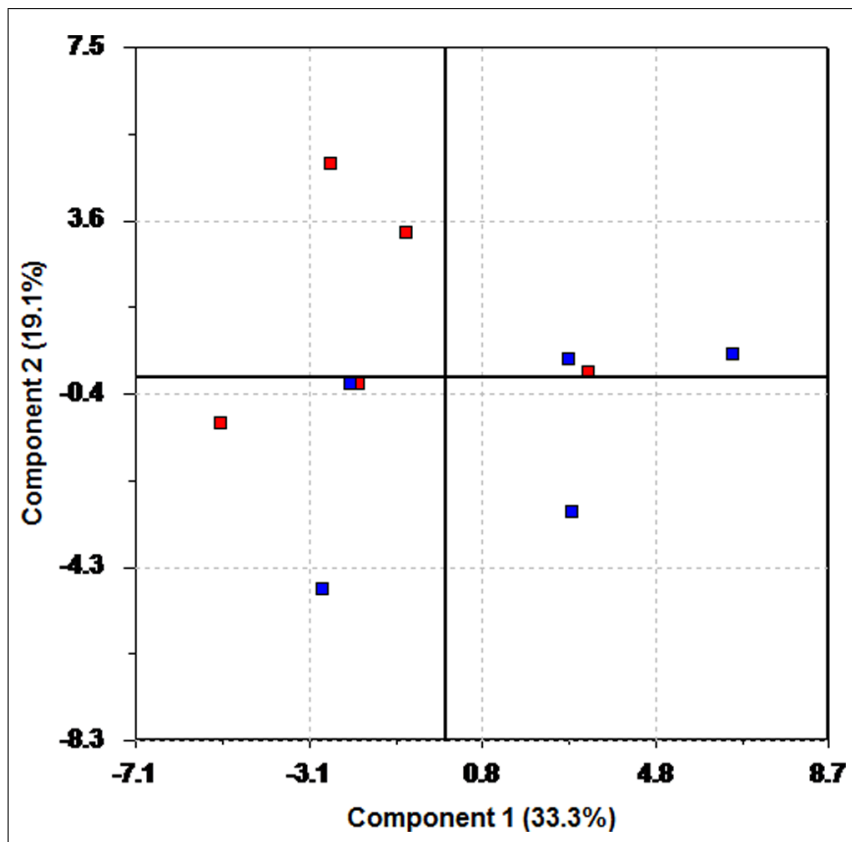

FIGURE 6 | Score plot of the first two principal components of the change data for the participants in the fish group having either a constant drug regime (blue) or receiving no medication (red).

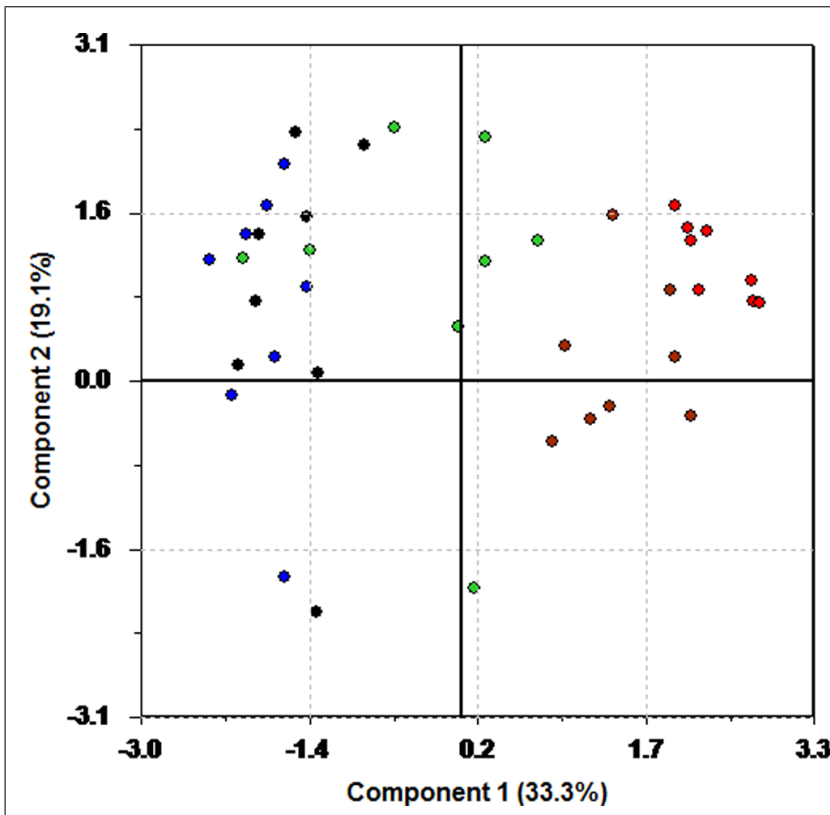

FIGURE 7 | Loading plot of the change data using only the participants in the fish group having a constant drug regime or receiving no medication. Red: HR. Blue: RMSSD. Green: LF. Black: HF. Brown: LF/HF.

results fit a previously observed pattern, where increased HRV was observed after exposure to a similar experimental mild-stress procedure (Hansen et al., 2003). Another study (Hansen and Johnsen, 2013) investigating the relationship between levels of neuroticism and performance on non-executive functioning tasks (tasks based on processes driven automatically), in both non-threatening and 
threatening situations, showed that participants with high neuroticism exposed to a threat had a significant increase in HRV from the task to recovery. This effect was absent when high neuroticism participants were not exposed to threats. For low neuroticism participants, this effect was absent regardless of threat level. In this latter study it was speculated whether the absence of threat in the recovery period caused the significant increase in HRV from test to recovery, since subjects with high neuroticism usually are characterized by low HRV. However, more research is needed to conclude whether information from the recovery measure can add something significant concerning mental and physical health.

Recently it was reported a strong relationship between tricyclic antidepressant and coronary heart disease (Kemp et al., 2015). Thus, as persons on heart medication and anti-depressants on average can be regarded as having poorer health (and thus lower life expectancy) than the general population, it is of interest to look deeper into the role of a fish diet for this group. The score and loading plots in Figures $\mathbf{6}$ and $\mathbf{7}$ generate the hypothesis that the participants using heart medication and/or anti-depressants throughout the fish intervention period experienced a larger reduction in LF than the ones not using drugs. Thus, considering the debate about the autonomic origins of LF power (Thayer et al., 2010; Xhyheri et al., 2012), the fact that $2 \mathrm{~min}$ data epochs provide better estimates of LF (Thayer et al., 2010), and the present findings, one could speculate whether the LF represents the sympathetic activity, and that intake of fatty fish may be even more beneficial for the participants on heart medication. However, there is empirical evidence that LF power correlate highly with HF power (Thayer and Lane, 2007). Because of this and the small sample size, further investigation is necessary.

As with many diet intervention studies, the number of participants in this study is low. This becomes even more evident if trying to control for the different types of medication in use. The number of participants is not large enough to warrant a more thorough analysis of the effects of the different medicines. One may also speculate as to whether the effect of the medicines may really be due to the underlying pathology, and not the medication. There is a debate concerning this relationship and a recent study focusing on depression demonstrated that reduction in HRV was related to pathophysiological mechanisms rather than the effect of antidepressant (SSRI; see Brunoni et al., 2013). In the present study, all participants diagnosed with CVD or depression was on medication. To discern between medication and pathology is therefore not possible using this data set. Still, there are numerous reports in the literature about the effects of various medications on HR and HRV, and the effect we observe in our study thus seems reasonable. The consistency in which the participants group when controlling for medication gives credence to the findings presented here. The focus on the change data and the subsequent explorative multivariate analysis is not meant to replace traditional statistics. We have also employed $t$-tests on the latent variables to indicate whether the observed groupings are reasonable. Used in this way, this approach represents a valuable addition to the data analytical tools normally employed.

The original motivation behind this intervention experiment was to look at the effect of the nutrition on mental health (e.g., Hansen et al., 2014a,b). The physical health benefits of introducing fatty fish to our diet has been amply demonstrated in earlier publications. Van Horn et al. (2008) present a thorough review of this dietary aspect with regards to CVDs. For institutions having a clientele with a lower than average life expectancy, this effect should be factored in when designing health improvement plans. The question that remains to be answered is whether increased fatty fish consumption can enhance the effect of traditional medical treatment such as antidepressants and heart medication and further increase the life expectancy. It has been reported that while anti-depressants reduce the symptoms of depression, they may leave the HRV at sub-optimal levels. As HRV is a strong predictor of cardiac status, this indicates that persons suffering from depression are vulnerable to CVDs. Thus, based on the present data exploration it can be hypothesized that the beneficial effects of an increased fatty fish intake are even larger for this group. More knowledge about this will have important implications with regards to the development of health improvement interventions in psychiatric institutions.

\section{AUTHOR CONTRIBUTIONS}

All authors have contributed to the design, analysis, interpretation, or acquisition. Drafting, revision, and final approval has been conducted by all authors. All authors agree to be accountable for all aspects of the work.

\section{ACKNOWLEDGMENTS}

The present study was supported by grants from the "Program board Nutrition, University of Bergen, Norway.” The authors wish to thank all participants for their cooperation. Thanks to Grethe Rosenlund at Skretting for providing the Atlantic salmon. We also wish to thank the kitchen staff for preparing all the meals and the health department for collecting blood samples at the secure forensic inpatient facility in US.

\section{REFERENCES}

Anda, R., Williamson, D., Jones, D., Macera, C., Eaker, E., Glassman, A., et al. (1993). Depressed affect, hopelessness, and the risk of ischemic heart disease in a cohort of US adults. Epidemiology 4, 285-294. doi: 10.1097/00001648-199307000-00003 Appelhans, B. M., and Luecken, L. J. (2006). Heart rate variability as an index of regulated emotional responding. Rev. Gen. Psychol. 10, 229-240. doi: 10.1037/1089-2680.10.3.229

Balogh, S., Fitzpatrick, D. F., Hendricks, S. E., and Paige, S. R. (1993). Increases in heart rate variability with successful treatment in patients with major depressive disorder. Psychopharmacol. Bull. 29, 201-206.

Barefoot, J. C., Helms, M. J., Mark, D. B., Blumenthal, J. A., Califf, R. M., Haney, T. L., et al. (1996). Depression and long-term mortality risk in patients with coronary artery disease. Am. J. Cardiol. 78, 613-617. doi: 10.1016/S0002-9149(96)00380-3

Bonaduce, D., Petretta, M., Ianniciello, A., Apicella, C., Cavallaro, V., and Marciano, F. (1997). Comparison of verapamil versus felodipine on heart rate variability after acute myocardial infarction. Am. J. Cardiol. 79, 564-569. doi: 10.1016/S0002-9149(96)00816-8

Brage, S., Brage, N., Franks, P. W., Ekelund, U., and Wareham, N. J. (2005). Reliability and validity of the combined heart rate and movement sensor actiheart. Eur. J. Clin. Nutr. 59, 561-570. doi: 10.1038/sj.ejcn.1602118

Brunoni, A. R., Kemp, A. H., Dantas, E. M., Goulart, A. C., Nunes, M. A., Boggio, P. S., et al. (2013). Heart rate variability is a trait marker of major depressive disorder: evidence from the sertraline vs. electric current therapy to treat depression clinical study. Int. J. Neuropsychopharmacol. 16, 1937-1949. doi: $10.1017 /$ S1461145713000497

Carney, R. M., Rich, M. W., Tevelde, A., Saini, J., Clark, K., and Jaffe, A. S. (1987). Major depressive disorder in coronary artery disease. Am. J. Cardiol. 60, 12731275. doi: 10.1016/0002-9149(87)90607-2 
Christensen, J. H., Skou, H. A., Fog, L., Hansen, V. E., Vesterlund, T., Dyerberg, J., et al. (2001a). Marine n-3 fatty acids, wine intake, and heart rate variability in patients referred for coronary angiography. Circulation 103, 651-657. doi: 10.1161/01.CIR.103.5.651

Christensen, J. H., Skou, H. A., Madsen, T., Tørring, I., and Schmidt, E. B. (2001b). Heart rate variability and $n-3$ polyunsaturated fatty acids in patients with diabetes mellitus. J. Intern. Med. 249, 545-552. doi: 10.1046/j.1365-2796.2001.00841.x

Cook, J. R., Bigger, J. T. Jr., Kleiger, R. E., Fleiss, J. L., Steinman, R. C., and Rolnitzky, L. M. (1991). Effect of atenolol and diltiazem on heart period variability in normal persons. J. Am. Coll. Cardiol. 17, 480-484. doi: 10.1016/S0735-1097(10)80119-6

Dobnig, H., Pilz, S., Scharnagl, H., Renner, W., Seelhorst, U., Wellnitz, B., et al. (2008). Independent association of low serum 25-hydroxyvitamin D and 1, 25-Dihydroxyvitamin D levels with all-cause and cardiovascular mortality. Arch. Intern. Med. 168, 1340-1349. doi: 10.1001/archinte.168 12.1340

Ebbehøj, E., Poulsen, P. L., Hansen, K. W., Knudsen, S. T., Mølgaard, H., and Mogensen, C. E. (2002). Effects on heart rate variability of metoprolol supplementary to ongoing ACE-inhibitor treatment in Type I diabetic patients with abnormal albuminuria. Diabetologia 45, 965-975. doi: 10.1007/s00125-0020869-7

Galski, T., Thornton, K. E., and Shumsky, D. (1990). Brain dysfunction in sex offenders. J. Offender Rehabil. 16, 65-80. doi: 10.1300/J076v16n01_04

Giovannucci, E., Liu, Y., Hollis, B. W., and Rimm, E. B. (2008). 25-Hydroxyvitamin $\mathrm{D}$ and risk of myocardial infarction in men. Arch. Intern. Med. 168, 1174-1180. doi: 10.1001/archinte.168.11.1174

Gomes, M. E., Tack, C. J., Verheugt, F. W., Smits, P., and Lenders, J. W. (2010). Sympathoinhibition by atorvastatin in hypertensive patients. Circulation 74, 2622-2626. doi: 10.1253/circj.CJ-10-0427

Gonzalez, M. B., Snyderman, T. B., Colket, J. T., Arias, R. M., Jiang, J. W., O'Connor, C. M., et al. (1996). Depression in patients with coronary artery disease. Depression 4, 57-62. doi: 10.1002/(SICI) 1522-7162(1996)4:2<57::AIDDEPR3>3.0.CO;2-C

Guedon-Moreau, L., Pinaud, A., Logier, R., Caron, J., Lekieffre, J., Dupuis, B., et al. (1997). Effect of ramipril on heart rate variability in digitalis-treated patients with chronic heart failure. Cardiovasc. Drugs Ther. 11, 531-536. doi: 10.1023/A: 1007763131888

Hansen, A. L., Dahl, L., Bakke, L., Frøyland, L., and Thayer, J. F. (2010). Fish consumption and heart rate variability. Preliminary results. J. Psychophysiol. 24, 41-47. doi: 10.1027/0269-8803/a000005

Hansen, A. L., Dahl, L., Olson, G., Thornton, D., Graff, I. E., Frøyland, L., et al. (2014a). Fish consumption, sleep, daily functioning, and heart rate variability. J. Clin. Sleep Med. 10, 567-575. doi: 10.5664/jcsm.3714

Hansen, A. L., Olson, G., Dahl, L., Thornton, D., Grung, B., Graff, I. E. et al. (2014b). Reduced anxiety in forensic inpatients after a long-term intervention with Atlantic salmon. Nutrients 6, 5405-5418. doi: 10.3390/nu61 25405

Hansen, A. L., and Johnsen, B. H. (2013). Relationship between neuroticism, threat of shock and HRV reactivity. Int. Marit. Health 64, 54-60.

Hansen, A. L., Johnsen, B. H., Sollers, J. J., Stenvik, K., and Thayer, J. F. (2004). Heart rate variability and its relation to prefrontal cognitive function: the effects of training and detraining. Eur. J. Appl. Physiol. 93, 263-272. doi: 10.1007/s00421004-1208-0

Hansen, A. L., Johnsen, B. H., and Thayer, J. F. (2003). Vagal influence on working memory and attention. Int. J. Psychophysiol. 48, 263-274. doi: 10.1016/S01678760(03)00073-4

Hansen, A. L., Johnsen, B. H., and Thayer, J. F. (2009). Relationship between heart rate variability and cognitive function during threat of shock. Anxiety Stres Coping 22, 1-12. doi: 10.1080/10615800802272251

Hibbeln, J. R., Ferguson, T. A., and Blasbalg, T. L. (2006). Omega-3 fatty acid deficiencies in neurodevelopment, aggression, and autonomic dysregulation: opportunities for intervention. Int. Rev. Psychiatry 18, 107-118. doi: 10.1080/09540260600582967

Huikuri, H., Raatikainen, M., Moerch-Joergensen, R., Hartikainen, J., Virtanen, V., Boland, J., et al. (2009). Prediction of fatal or near-fatal cardiac arrhythmia events in patients with depressed left ventricular function after an acute myocardial infarction. Eur. Heart J. 30, 689-698. doi: 10.1093/eurheartj/ehn537

Jackson, J. E. (1991). A User's Guide To Principal Components. New York: John Wiley \& Sons, Inc. doi: 10.1002/0471725331
Joliffe, I. T. (2002). Principal Component Analysis. New York: Springer.

Kemp, A. H., Brunoni, A. R., Bittencourt, M. S., Nunes, M. A., Benseñor, I. M., and Lotufo, P. A. (2015). The association between antidepressant medications and coronary heart disease in Brazil: a cross-sectional analysis on the Brazilian longitudinal study of adult health (ELSA-Brazil). Front. Public Health 3:9. doi: 10.3389/fpubh.2015.00009

Kemp, A. H., Brunoni, A. R., Santos, I. S., Nunes, M. A., Dantas, E. M., de Figueiredo, R. C., et al. (2014). Effects of depression, anxiety, comorbidity, and antidepressants on resting-state heart rate and its variability: an ELSA-Brasil cohort baseline study. Am. J. Psychiatry doi: 10.1176/appi.ajp.2014.13121605 [Epub ahead of print].

Kemp, A. H., Quintana, D. S., Gray, M. A., Felmingham, K. L., Brown, K., and Gatt, J. M. (2010). Impact of depression and antidepressant treatment on heart rate variability: a review and meta-analysis. Biol. Psychiatry 67, 1067-1074. doi: 10.1016/j.biopsych.2009.12.012

Khaykin, Y., Dorian, P., Baker, B., Shapiro, C., Sandor, P., Mironov, D., et al. (1998). Autonomic correlates of antidepressant treatment using heart-rate variability analysis. Can. J. Psychiatry 43, 183-186.

Kleiger, R. E., Stein, P. K., and Bigger, J. T. (2005). Heart rate variability: measurement and clinical utility. Ann. Noninvasive Electrocardiol. 10, 88-101. doi: 10.1111/j.1542-474X.2005.10101.x

Lansdowne, A. T. G., and Provost, S. C. (1998). Vitamin D3 enhances mood in healthy subjects during winter. Psychopharmacology (Berl.) 135, 319-323. doi: $10.1007 / \mathrm{s} 002130050517$

Licht, C. M., de Geus, E. J., van Dyck, R., and Penninx, B. W. (2010). Longitudinal evidence for unfavorable effects of antidepressants on heart rate variability. Biol. Psychiatry 68, 861-868. doi: 10.1016/j.biopsych.2010. 06.032

Malik, M., and Task Force of the European Society of Cardiology the North American Society of Pacing Electrophysiology. (1996). Heart rate variability. Standards of measurement, physiological interpretation, and clinical use. Circulation 93, 1043-1065. doi: 10.1161/01.CIR.93.5.1043

Malliani, A., Pagani, M., Lombardi, F., and Cerutti, S. (1991). Cardiovascular neural regulation explored in the frequency-domain. Circulation 84, 482-492. doi: 10.1161/01.CIR.84.2.482

Manne, R. (1987). Analysis of two partial-least-squares algorithms for multivariate calibration. Chemometr. Intell. Lab. Syst. 2, 187-197. doi: 10.1016/01697439(87)80096-5

Melenovsky, M., Wichterle, D., Simek, J., Malik, J., Haas, T., Ceska, R., et al. (2003). Effect of atorvastatin and fenofibrate on autonomic tone in subjects with combined hyperlipidemia. Am. J. Cardiol. 92, 337-341. doi: 10.1016/S0002-9149(03)00643-X

Mølgaard, H., Mickley, H., Pless, P., Bjerregaard, P., and Møller, M. (1993). Effects of metoprolol on heart rate variability in survivors of acute myocardial infarction. Am. J. Cardiol. 71, 1357-1359. doi: 10.1016/0002-9149(93) 90555-Q

Mozaffarian, D., Stein, P. K., Prineas, R. J., and Siscovick, D. S. (2008). Dietary fish $\omega-3$ fatty acid consumption and heart rate variability in US adults. Circulation 117, 1130-1137. doi: 10.1161/CIRCULATIONAHA.107. 732826

Niemelä, M. J., Airaksinen, K. E., and Huikuri, H. V. (1994). Effect of beta-blockade on heart rate variability in patients with coronary artery disease. J. Am. Coll. Cardiol. 23, 1370-1377. doi: 10.1016/0735-1097(94)90379-4

Nome, S., and Holsten, F. (2012). Changes in mortality after first psychiatric admission: a 20-year prospective longitudinal clinical study. Nord. J. Psychiatry 66, 97-106. doi: 10.3109/08039488.2011.605170

Partonen, T. (1998). Vitamin D and serotonin in winter. Med. Hypotheses 51, 267268. doi: 10.1016/S0306-9877(98)90085-8

Penninx, B. W., Beekman, A. T., Honig, A., Deeg, D. J., Schoevers, R. A., van Eijk, J. T., etal. (2001). Depression and cardiac mortality: results from a community-based longitudinal study. Arch. Gen. Psychiatry 58, 221-227. doi: 10.1001/archpsyc.58.3.221

Perica, M. M., and Delas, I. (2011). Essential fatty acids and psychiatric disorders. Nutr. Clin. Pract. 26, 409-425. doi: 10.1177/0884533611411306

Pinar, E., Garcia-Alberola, A., Llamas, C., Vicente, T., Lopez-Candel, J., Rojo, J. L., etal. (1998). Effects of verapamil on indexes of heart rate variability after acute myocardial infarction. Am. J. Cardiol. 81, 1085-1089. doi: $10.1016 /$ S0002-9149(98)00128-3 
Porges, S. W., and Raskin, D. C. (1969). Respiratory and heart rate components of attention. J. Exp. Psychol. 81, 497-503. doi: 10.1037/h0027921

Pratt, L. A., Ford, D. E., Crum, R. M., Armenian, H. K., Gallo, J. J., and Eaton, W. W. (1996). Depression, psychotropic medication, and risk of myocardial infarction. Prospective data from the Baltimore ECA follow-up. Circulation 94, 3123-3129. doi: 10.1161/01.CIR.94.12.3123

Quintana, D. S., and Heathers, J. (2014). Considerations in the assessment of heart rate variability in biobehavioral research. Front. Psychol. 5:805. doi: 10.3389/fpsyg.2014.00805

Riahi, S., Christensen, J. H., Toft, E., Skou, H. A., and Schmidt, E. B. (2002). HMG-CoA reductase inhibitors improve heart rate variability in patients with a previous myocardial infarction. Pharmacol. Res. 45, 479-483. doi: 10.1006/phrs. 2002.0988

Ross, B. M., Seguin, J., and Sieswerda, L. E. (2007). Omega-3 fatty acids as treatments for mental illness: which disorder and which fatty acid? Lipids Health Dis. 6, 1-19. doi: 10.1186/1476-511X-6-21

Sandrone, G., Mortara, A., Torzillo, D., La Rovere, M. T., Malliani, A., and Lombardi, F. (1994). Effects of beta blockers (atenolol or metoprolol) on heart rate variability after acute myocardial infarction. Am. J. Cardiol. 74, 340-345. doi: 10.1016/0002-9149(94)90400-6

Shehab, A., Elnour, A. A., and Struthers, A. D. (2008). A randomised, controlled, double-blind, cross-over pilot study assessing the effects of spironolactone, losartan and their combination on heart rate variability and QT dispersion in patients with chronic heart failure. Cardiovasc. J. Afr. 19, 292-296.

Shimamura, A. P. (2000). The role of the prefrontal cortex in dynamic filtering Psychobiology 28, 207-218. doi: 10.3758/BF03331979

Stumpf, W. E., and Privette, T. H. (1989). Light, vitamin D and psychiatry: role of 1,25- dihydroxyvitamin D3 (soltriol) in etiology and therapy of seasonal affective disorder and other mental processes. Psychopharmacology (Berl.) 97, 285-294. doi: 10.1007/BF00439440

Thayer, J. F., Hansen, A. L., and Johnsen, B. H. (2010). “The non-invasive assessment of autonomic influences on the heart using impedance cardiography and heart rate variability," in Handbook of Behavioural Medicine - Methods and Applications, ed. A. Steptoe (London: Springer), 723-740.

Thayer, J. F., and Lane, R. D. (2000). A model of neurovisceral integration in emotion regulation and dysregulation. J. Affect. Disord. 61, 201-216. doi: 10.1016/S01650327(00)00338-4

Thayer, J. F., and Lane, R. D. (2007). The role of vagal function in the risk for cardiovascular disease and mortality. Biol. Psychol. 74, 224-242. doi: 10.1016/j.biopsycho.2005.11.013
Thayer, J. F., and Lane, R. D. (2009). Claude Bernard and the heart-brain connection: further elaboration of a model of neurovisceral integration. Neurosci. Biobehav. Rev. 33, 81-88. doi: 10.1016/j.neubiorev.2008.08.004

Timonen, M., Horrobin, D., Jokelainen, J., Laitinen, A. H., and Räsänen, P. (2004). Fish consumption and depression: the Northern Finland 1966 birth cohort study. J. Affect. Disord. 82, 447-452. doi: 10.1016/j.jad.2004.02.002

Van Horn, L., McCoin, M., Kris-Etherton, P. M., Burke, F., Carson, J. A., Champagne, C. M., etal. (2008). The evidence for dietary prevention and treatment of cardiovascular disease. J. Am. Diet. Assoc. 108, 287-331. doi: 10.1016/j.jada.2007.10.050

van Zyl, L. T., Hasegawa, T., and Nagata, K. (2008). Effects of antidepressant treatment on heart rate variability in major depression: a quantitative review. Biopsychosoc. Med. 2, 12 doi: 10.1186/1751-0759-2-12

Welzig, C. M., Shin, D. G., Park, H. J., Kim, Y. J., Saul, J. P., and Galper, J. N. (2003). Lipid lowering by pravastatin increases parasympathetic modulation of heart rate: Galpha(i2), a possible molecular marker for parasympathetic responsiveness. Circulation 108, 2743-2746. doi: 10.1161/01.CIR.0000103680.61390.16

Xhyheri, B., Manfrini, O., Mazzolini, M., Pizzi, C., and Bugiardini, R. (2012). Heart rate variability today. Prog. Cardiovasc. Dis. 55, 321-331. doi: 10.1016/j.pcad.2012.09.001

Conflict of Interest Statement: The authors declare that the research was conducted in the absence of any commercial or financial relationships that could be construed as a potential conflict of interest.

Received: 29 September 2014; accepted: 26 January 2015; published online: 17 February 2015.

Citation: Grung B, Hansen AL, Berg M, Møen-Knudseth MP, Olson G, Thornton D, Dahl L and Thayer JF (2015) Exploratory multivariate analysis of the effect of fatty fish consumption and medicinal use on heart rate and heart rate variability data. Front. Psychol. 6:135. doi: 10.3389/fpsyg.2015.00135

This article was submitted to Emotion Science, a section of the journal Frontiers in Psychology.

Copyright (C) 2015 Grung, Hansen, Berg, Møen-Knudseth, Olson, Thornton, Dahl and Thayer. This is an open-access article distributed under the terms of the Creative Commons Attribution License (CC BY). The use, distribution or reproduction in other forums is permitted, provided the original author(s) or licensor are credited and that the original publication in this journal is cited, in accordance with accepted academic practice. No use, distribution or reproduction is permitted which does not comply with these terms. 